ARTICLE

Received 3 Jun 2014 | Accepted 24 Sep 2014 | Published 5 Nov $2014 \quad$ DOl: 10.1038/ncomms6377

\title{
Effector CD4 T-cell transition to memory requires late cognate interactions that induce autocrine IL-2
}

K. Kai McKinstry ${ }^{1, \star}$, Tara M. Strutt ${ }^{1, \star}$, Bianca Bautista ${ }^{1}$, Wenliang Zhang ${ }^{1}$, Yi Kuang $^{1}$, Andrea M. Cooper ${ }^{2} \&$ Susan L. Swain ${ }^{1}$

It is unclear how CD4 T-cell memory formation is regulated following pathogen challenge, and when critical mechanisms act to determine effector T-cell fate. Here, we report that following influenza infection most effectors require signals from major histocompatibility complex class II molecules and CD70 during a late window well after initial priming to become memory. During this timeframe, effector cells must produce IL-2 or be exposed to high levels of paracrine or exogenously added IL-2 to survive an otherwise rapid default contraction phase. Late IL-2 promotes survival through acute downregulation of apoptotic pathways in effector T cells and by permanently upregulating their IL-7 receptor expression, enabling IL-7 to sustain them as memory T cells. This new paradigm defines a late checkpoint during the effector phase at which cognate interactions direct CD4 T-cell memory generation.

\footnotetext{
${ }^{1}$ Department of Pathology, University of Massachusetts Medical School, 55 Lake Avenue North, Worcester, Massachusetts 01655, USA. ${ }^{2}$ Trudeau Institute, 154 Algonquin Avenue, Saranac Lake, New York 12983, USA. * These authors contributed equally to this work. Correspondence and requests for materials should be addressed to K.K.M. (email: kai.mckinstry@umassmed.edu).
} 
nfection with viruses and other rapidly replicating pathogens generates large numbers of effector CD4 T cells in secondary lymphoid sites, where they originate, and at sites of infection to which they migrate. As the pathogen is cleared, most effectors abruptly die, leaving a small cohort that transition to long-lived memory ${ }^{1,2}$. It is unclear to what extent the contraction of effectors and transition of surviving cells to memory are programmed during early encounter with antigen-presenting cells (APC) during priming ${ }^{3}$ and/or by external factors triggered by infection acting at later stages of the response. As the CD4 T-cell response to influenza A virus (IAV) generates memory cells capable of clearing heterosubtypic IAV challenge ${ }^{4,5}$, it is a wellsuited model for defining the mechanisms regulating the efficiency of CD4 T-cell memory generation.

One suspected cause of T-cell contraction is 'cytokine withdrawal' that is triggered by inadequate access to growth and survival factors such as IL-2 that limit apoptosis ${ }^{6}$. After initial stimulation, naive CD4 T cells make IL-2, which causes the cells to differentiate and may also support their survival and division. Early IL-2 can also programme responding $\mathrm{T}$ cells to have an increased capacity for memory survival and function ${ }^{7-9}$. However, the effect of later IL-2 signalling is less clear. On the one hand, exposure to IL-2 during the expansion phase can drive enhanced sensitivity to apoptosis ${ }^{10,11}$ and to re-stimulation-induced cell death $^{12}$. In contrast, our previous studies indicated that CD4 $\mathrm{T}$-cell effectors generated in vitro were programmed to die and that IL-2 (plus transforming growth factor beta) could block their apoptosis $^{13}$. This mechanism of effector T-cell rescue has not been thoroughly evaluated for a role in memory generation in vivo.

We considered that late cognate interactions might promote memory formation. This would be attractive as such regulation could link a continuing threat from a pathogen with the level of memory generated. We reasoned that differentiated effector cells responding to IAV might be induced by APC to make IL- 2 that could prevent their acute apoptosis and thus provide a key late 'checkpoint' governing the efficiency with which the effectors transition to long-lived memory.

We found that late major histocompatibility complex II (MHC-II) recognition in vivo from 4 to 6 days post-infection (d.p.i.), as CD4 T-cell responses against IAV reach their peak ${ }^{14}$, indeed promoted greater recovery of memory cells during primary and secondary responses. Autocrine IL-2 production by effectors, or high levels of exogenously administered IL-2, during this timeframe was required for the generation of almost all memory cells. This late IL-2 signalling rescued effectors from acute apoptosis and upregulated sustained CD127 expression. The level of enhanced CD127 expression correlated directly with the amount of late IL-2 available and with the size of the memory population generated. Finally, late signals from CD70, which act through CD27 expressed on effector CD4 T cells to enhance IL-2 during cognate recognition, were required for optimal memory generation. Our results define a novel late checkpoint at which CD4 T-cell effectors must engage in cognate interactions to induce autocrine IL-2 that signals them to survive and is necessary for them to become long-lived memory cells.

\section{Results}

Memory is reduced by MHC-II blockade at the effector stage. To evaluate whether cognate interactions of CD4 T-cell effectors with $\mathrm{MHC}-\mathrm{II}^{+}$APC are needed to promote memory generation, we asked whether blocking MHC-II with antibody (Ab) treatment ${ }^{15}$ only at the effector stage would reduce memory cell recovery following IAV challenge. To avoid complications arising from the differential ability of cells with different T-cell receptors (TcR) to form memory ${ }^{16,17}$, and the predicted lack of synchrony

in polyclonal responses, we tracked small cohorts of adoptively transferred TcR transgenic (Tg) cells. As it is likely that even extremely large doses of monoclonal $\mathrm{Ab}$ would not efficiently block MHC-II expression in wild-type (WT) mice throughout the effector stage, we utilized as hosts CD11cTg.H2-Ab1 ${ }^{-/-}$mice that only express MHC-II on $\mathrm{CD}_{11 \mathrm{c}^{+}}$cells $^{18}$. We first transferred naive OT-II cells to $\mathrm{C} 57 \mathrm{BL} / 6$ or CD11cTg.H2$A b 1^{-/-}$mice and challenged with a sublethal dose of the recombinant A/PuerotRico/8/34-Ovalbumin $323-339$ (PR8-OVA $\left.{ }_{\text {II }}\right)$ virus that contains the OVA epitope recognized by the OT-II $\mathrm{TcR}^{19}$. Donor cell recovery at 7 and 28 d.p.i. was equivalent in both hosts, indicating that MHC-II expression restricted to CD11c ${ }^{+}$cells was sufficient for optimal effector expansion and efficient memory generation (Fig. 1a). Importantly, treatment of

a

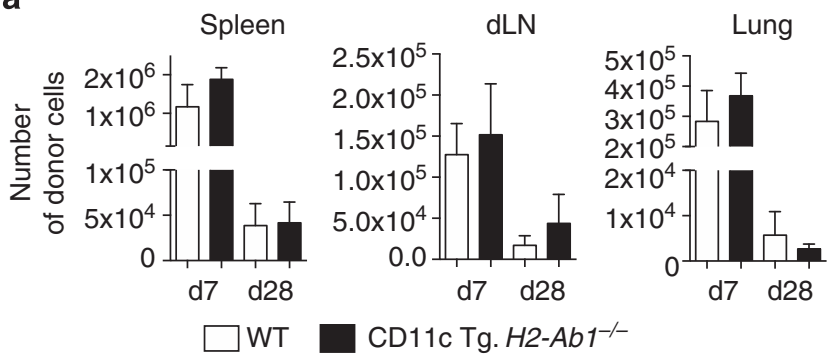

b

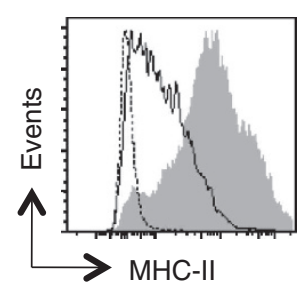

C

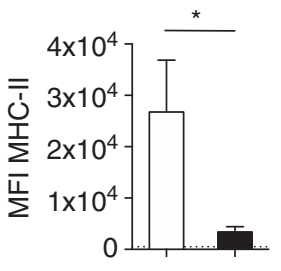

Isotype

Isotype

Anti-MHC Class II blocking Ab

Anti-MHC Class II blocking Ab

d

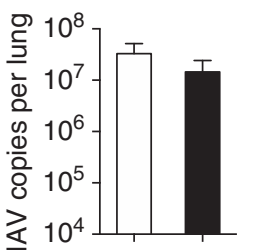

$\square$ Isotype

Anti-MHC Class II blocking Ab

Figure 1 | Blocking interactions with MHC-II + APC at the effector stage reduces memory CD4 T cell generation following IAV challenge. (a) Recovery of WT donor cells in the spleen, draining lymph node (dLN) and lung at 7 d.p.i., the peak of effector response, and 28 d.p.i., a memory time point, in WT C57BL/6 and CD11cTg.H2-Ab1-/- hosts challenged with IAV. CD11c Tg.H2-Ab1 -/- recipients of WT donor cells challenged with IAV were treated with isotype or anti-MHC-II blocking Ab on 4-6 d.p.i. (four mice per group; one of two experiments). (b,c) Representative staining and mean fluorescent intensity (MFI) of $\mathrm{MHC}-\mathrm{II}$ on $\mathrm{CD}_{11 \mathrm{c}}{ }^{+}$cells on 7 d.p.i. Negative MHC-II staining of CD11c ${ }^{-}$cells is shown as a dashed line. (d) Viral titer on 7 d.p.i. and (e) recovery of naive and in vitro-primed Th1-polarized memory (primed) donor cells at 7 and 28 d.p.i. following isotype or anti-MHC-II Ab treatment from 4 to 6 d.p.i. (four mice per group; one of two experiments). All error bars represent the s.d. and significant differences determined with unpaired, two-tailed, Student's $t$-tests $\left(\alpha=0.05\right.$ and $\left.{ }^{\star} P<0.05,{ }^{\star \star} P<0.005\right)$. 
CD11cTg.H2-Ab1 ${ }^{-/-}$mice with MHC-II blocking Ab from 4 to 6 d.p.i. markedly reduced MHC-II expression on CD11 $\mathrm{c}^{+}$cells as assessed by flow cytometry at 7 d.p.i. (Fig. 1b,c).

We next tested the effect of MHC-II blockade from 4 to 6 d.p.i. in CD11cTg.H2-Ab1 ${ }^{-/-}$hosts on OT-II donor cell expansion and memory generation. We used naive as well as in vitro-primed $\mathrm{T}_{\mathrm{H}}$ 1-polarized memory cells, which respond against IAV similarly to IAV-primed memory cells ${ }^{4,14}$, as donors to ask whether secondary memory generation, such as following a vaccine boost, was also dependent on late MHC-II recognition. $\mathrm{Ab}$ treatment from 4 to 6 d.p.i. did not impact viral titre detected at 7 d.p.i. (Fig. 1d). MHC-II blockade did reduce primary effector expansion in the lung at 7 d.p.i. but did not impact secondary effector responses (Fig. 1e). More importantly, late MHC-II blockade markedly reduced donor cell recovery at 28 d.p.i. in recipients of naive and primed cells (Fig. 1e), suggesting that cognate antigen $(\mathrm{Ag})$ recognition during the effector phase is needed for efficient memory formation during primary and secondary CD4 T-cell responses.

Generation of memory is impaired in the absence of IL-2. We used several strategies to determine whether the effector cells require late IL-2 signals that were potentially induced by MHCII-dependent interactions to efficiently transition to memory. First, we transferred naive WT or $I 2^{-/-}$DO11.10 cells to WT $\mathrm{BALB} / \mathrm{c}$ hosts and challenged with PR8-OVA $\mathrm{II}$. Compared with WT donors, $I l 2^{-/-}$cells reached lower peak numbers (Fig. 2a), consistent with a requirement for IL-2 for optimal primary expansion in $v i v o^{20-22}$. More notably the $I / 2^{-1-}$ effectors underwent more rapid and extensive contraction than WT cells, resulting in little memory cell recovery. Interestingly, the number of $I l 2^{-9}$ - donor cells seemed to stabilize briefly between 10 and 14 d.p.i. Nevertheless, in the spleen and draining lymph node (dLN), $I l 2^{-/-}$cells became virtually non-existent by 28 d.p.i., whereas in the lung a significantly reduced population survived (Fig. 2a).

Utilizing naive $I 2^{-/-}$donor cells cannot distinguish whether the need for IL-2 for memory formation is late, during the timeframe when effectors required MHC-II-restricted encounter with $\mathrm{CD}_{11 \mathrm{c}^{+}}$cells (Fig. 1), and/or during initial priming ${ }^{13}$. Therefore, we examined responses of $\mathrm{Il}^{-9}$ in vitro-primed memory cells, generated with exogenously added IL-2, reasoning that these cells would have received initial IL-2 programing required for memory competency ${ }^{9}$, and that their effector survival might only require IL-2 late during the response against IAV. Indeed, both WT and $I l 2^{-1-}$ in vitro-primed memory cells generated with exogenous IL-2 rapidly produced IFN- $\gamma$ (Supplementary Fig. 1), expressed a prototypical resting memory phenotype and survived long-term on transfer to uninfected WT hosts (Supplementary Fig. 2). Thus, priming with exogenous IL-2 in vitro generated long-lived functional $\mathrm{Il} 2^{-/-}$memory cells.

We compared responses and survival of adoptively transferred IL-2-primed WT or $I l 2^{-/-}$donors following IAV challenge. Both populations generated similar peak numbers of secondary effectors (Fig. 2b) that expressed an activated phenotype and produced high levels of IFN- $\gamma$ and TNF (Supplementary Fig. 3). The number of $I 2^{-/-}$donor cells fell sharply between 7 and 10 d.p.i. and, as observed with unprimed $I 2^{-1}-$ donors, appeared to stabilize briefly between 10 and 14 d.p.i. in the dLN and spleen (Fig. 2b). Strikingly, the $I 2^{-1-}$ donor cells contracted much more markedly than WT cells and were barely detectable at 28 d.p.i. in the spleen and dLN with many fewer cells surviving than WT donors in the lung (Fig. 2b), reproducing the pattern seen with naive donors (Fig. 2a).
To control for artefacts that could result from the deletion of IL-2 throughout CD4 T-cell development, we transferred WT in vitro-generated memory cells and treated recipients with IL-2 neutralizing Abs from 1 to 7 d.p.i. Blocking IL-2 did not affect donor cell recovery at 7 d.p.i., but it significantly reduced memory recovery in the lung and nearly eliminated memory in the spleen and dLN, phenocopying results obtained with $\mathrm{Il}^{-}{ }^{-}-$donor cells (Fig. 2c). Moreover, when IL-2 neutralizing Ab treatment was restricted to 4-7 d.p.i., memory was impaired to a nearly equal extent as with longer treatment from 1 to 7 d.p.i. (Fig. 2c). Importantly, no differences in viral titre were detected on 7 d.p.i. between isotype and IL-2 neutralizing Ab-treated mice (Fig. 2d). These data confirm that late IL-2 signals during the effector phase of the CD4 T-cell response are required for optimal memory generation.

IL-2 neutralization also reduced long-term survival of in vivogenerated polyclonal memory CD4 T cells responding to IAV. We transferred bulk 6-carboxyfluorescein succinimidyl ester (CFSE)-labelled CD4 T cells from IAV-primed WT mice to unprimed Thy-disparate hosts and infected with IAV. We identified IAV-specific donor cells using an IAV-specific MHCII-tetramer (Fig. 2e). IL-2 neutralization significantly reduced donor CFSE ${ }^{\text {low }}$ tetramer-positive polyclonal donor cells at 28 d.p.i. (Fig. 2f). Together these results suggest that following IAV infection CD4 T-cell-produced IL-2 is required to limit contraction of effectors and support their survival as memory cells.

High levels of IL-2 are needed to induce memory CD4 T cells. The experiments summarized in Fig. 2 suggest that autocrine IL-2 signals are needed to rescue effector $\mathrm{CD} 4 \mathrm{~T}$ cells from enhanced contraction as the number of $I 2^{-/-}$donor cells present at 28 d.p.i. in untreated hosts was similar to the number of WT donor cells present in hosts treated with anti-IL-2 Abs. To formally test whether autocrine IL-2 signalling is required to rescue effector cell survival, we cotransferred equal numbers of WT and $I l 2^{-/-}$ DO11.10 donor cells distinguishable by allelic markers (Fig. 3a). Cotransfer only slightly enhanced the poor survival of $I l 2^{-1-}$ cells (Fig. 3b). The simplest interpretation of these results is that memory generation is directly dependent on availability to effector cells of high levels of IL-2 that are likely present during physiological immune responses only when there is autocrine production. However, the slight improvement in survival of $I 2^{-1}$

- donors on cotransfer with WT cells suggests that high levels of paracrine IL-2 can enhance the long-term survival of $I 2^{-/-}$ $\mathrm{CD} 4 \mathrm{~T}$ cells responding against IAV.

To test whether the reduced survival of $I l 2^{-/-}$versus WT donor cells would result in an impaired recall response on the population level, or the lower number of surviving $I 2^{-/-}$donor cells was still sufficient to mount a robust secondary response, we challenged PR8-OVA ${ }_{\text {II }}$-primed recipients of WT and $I l 2^{-1-}$ donor cells with a heterosubtypic IAV virus expressing the $\mathrm{OVA}_{323-339}$ peptide $\left(\mathrm{X} 31-\mathrm{OVA}_{\mathrm{II}}\right) 30$ days post-priming. Both donor populations in the lung increased in forward scatter, indicating an increase in size and upregulated CD69 at 2 d.p.i. (Fig. 3c,d), but by 6 d.p.i. WT cells outnumbered $I l 2^{-/-}$donors by about 11-fold in the lung (Fig. 3e); while in the spleen and dLN WT cells were recovered but virtually no $\mathrm{Il}^{-1-}$ cells were detected (not shown). Thus, the impaired memory CD4 T-cell formation in the absence of autocrine IL-2 signalling resulted in a recall response of dramatically reduced magnitude.

Late administration of IL-2 substitutes for autocrine IL-2. To confirm that the lack of late IL-2 was responsible for the poor survival of $I 2^{-/-}$donor cells, we administered IL-2: anti-IL-2 $\mathrm{Ab}$ complexes ${ }^{23}$ (IL-2C) to recipients of primed donor cells, 

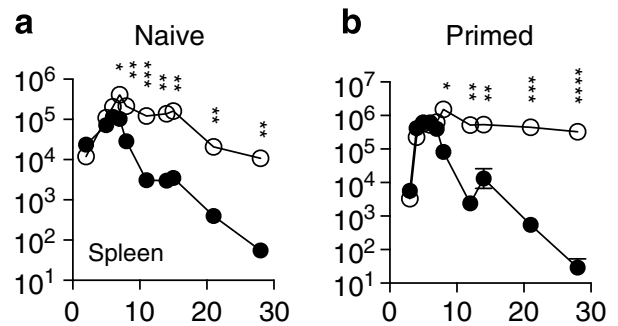

C
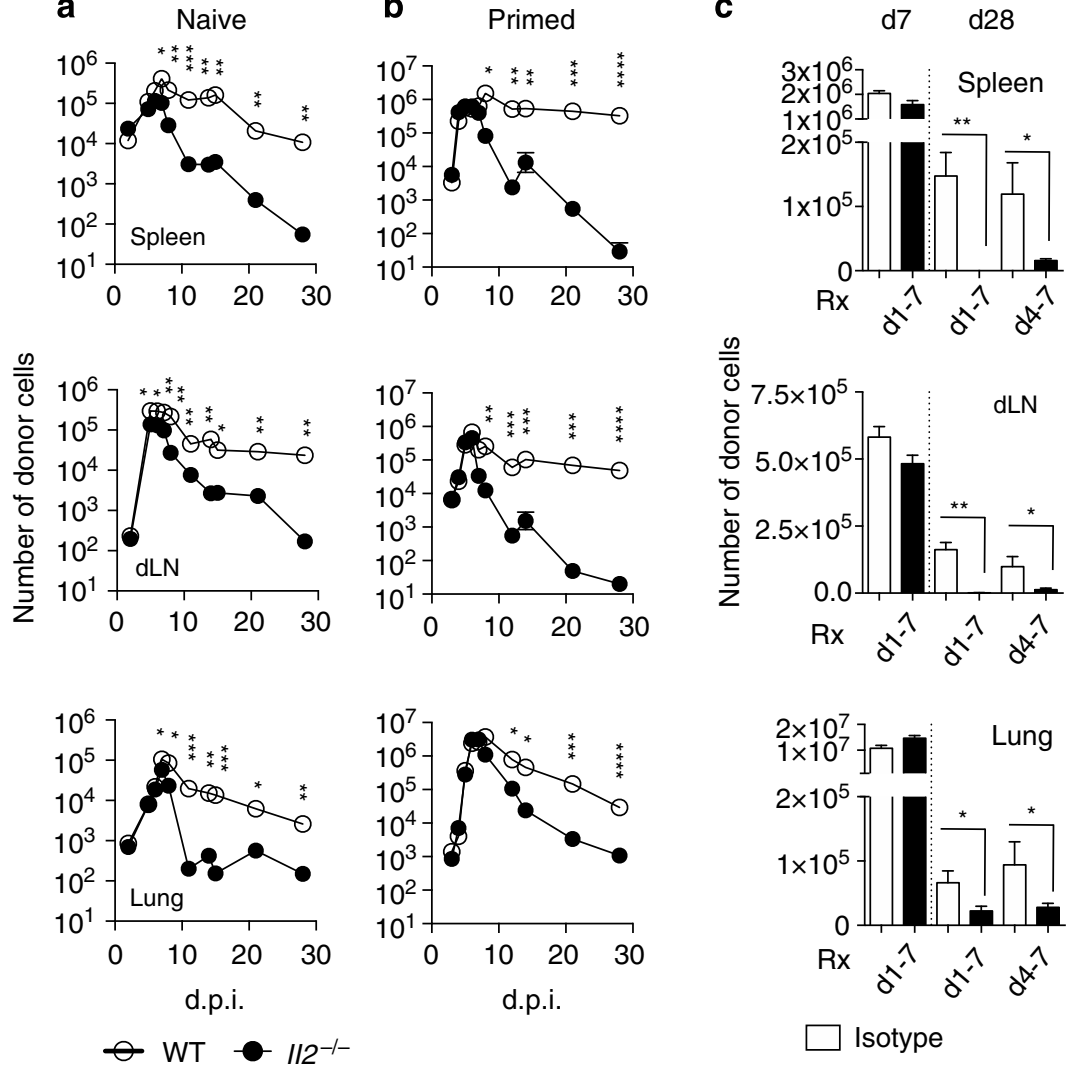

$\square$ Isotype

d

e

IL-2 neutralizing $A b$
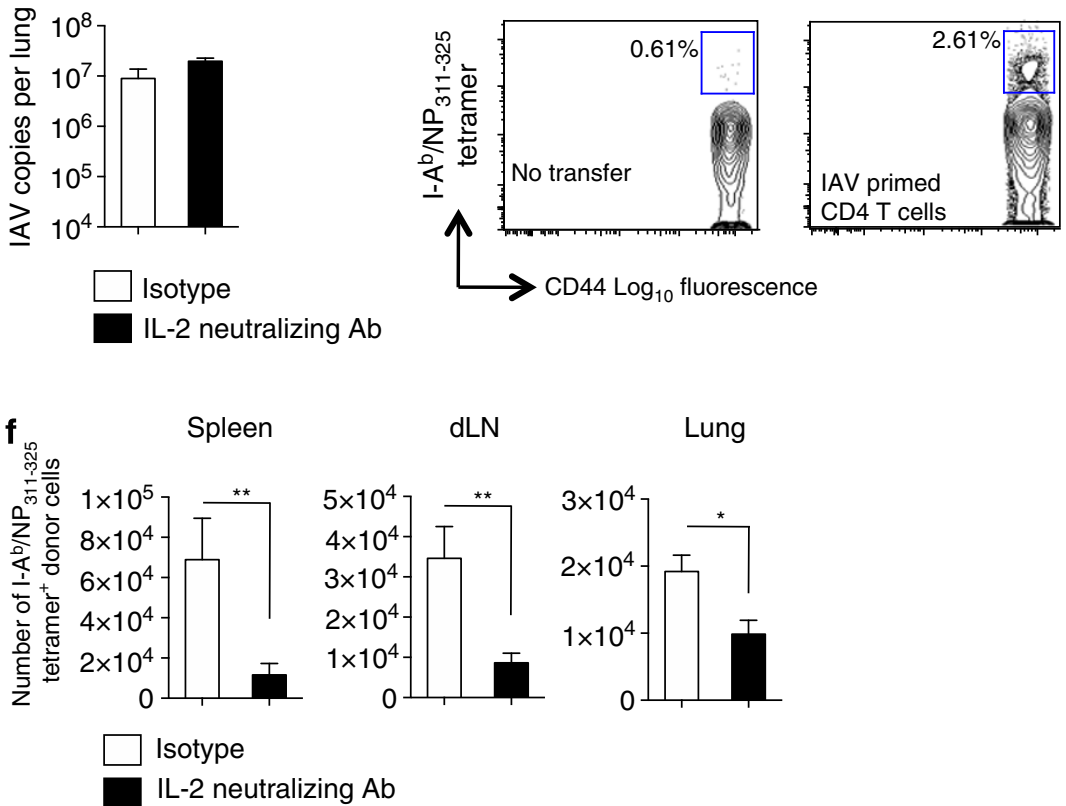

Figure 2 | IL-2 production is needed for IAV-specific memory CD4 T-cell generation. Unprimed BALB/c hosts received $1 \times 10^{6}$ congenically marked WT or $112^{-/-}$(a) naive or (b) primed DO11.10 cells. Donor cells were enumerated on the indicated days following IAV challenge (summary of at least two experiments per time point with at least three mice per group). Recipients of WT primed donors were treated with $250 \mu \mathrm{g}$ of each IL-2 neutralizing Ab from 1 to 7 d.p.i. or with $500 \mu$ g of each from 4 to 7 d.p.i. and (c) donors enumerated on 7 and 28 d.p.i. and (d) viral titres assessed on 7 d.p.i. (3-5 mice per group per day; 1 of 3 experiments). Polyclonal CD4 T cells from IAV-primed C57BL/ 6 mice were CFSE-labelled and $1 \times 10^{7}$ transferred to Thy-disparate hosts then challenged with IAV and treated with IL-2 neutralizing Ab from 1 to 7 d.p.i. At 28 d.p.i., tetramer-positive cells (e), within the CFSE ${ }^{\text {low }}$ donor population were enumerated in the spleen, $\mathrm{dLN}$ and lung (f) (summary of at least two experiments with at least three mice per group). All error bars represent the s.d. and significant differences determined with unpaired, two-tailed, Student's $t$-tests $\left(\alpha=0.05\right.$ and ${ }^{\star} P<0.05,{ }^{\star \star} P<0.005,{ }^{\star \star \star} P<0.001$, $\star \star \star \star * P<0.0001)$. 


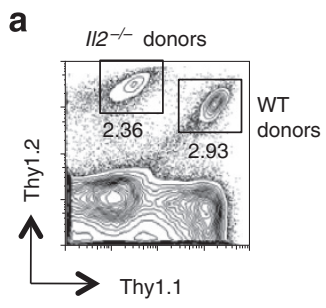

$\log _{10}$ fluorescence

C

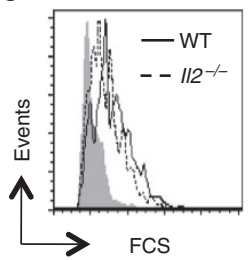

b

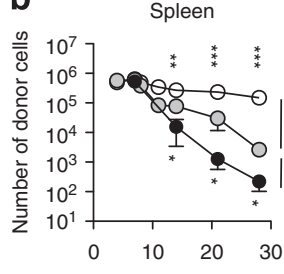

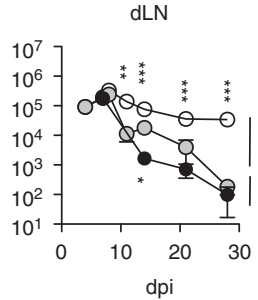

dpi

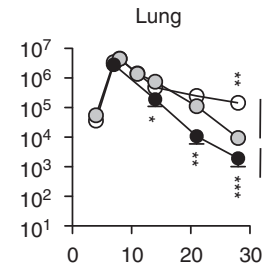

$\vartheta$ WT in cotransfer

-O- $112^{-/-}$in cotransfer

- $112^{-/-}$only
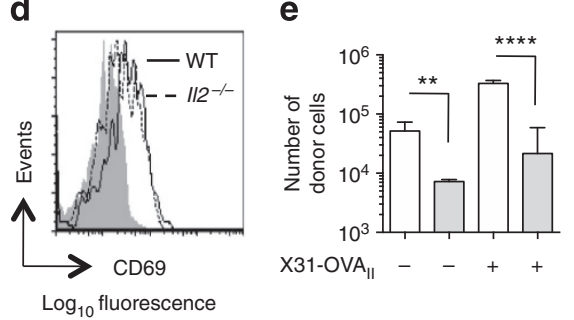

WT in cotransfer

$112^{-/-}$in cotransfer

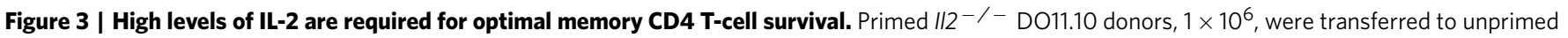
hosts either alone or together with $1 \times 10^{6}$ primed WT D011.10 cells and subsequently challenged with IAV. (a) In cotransferred mice, donors could be distinguished by Thy expression. (b) Donor populations were enumerated on stated d.p.i.: WT versus $/ 12^{-} /{ }^{-}$comparisons shown above and $/ 12^{-} /-$

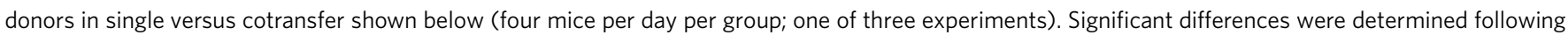
one-way ANOVA analysis with Bonferroni's multiple comparison post-test $\left.{ }^{\star} P<0.05,{ }^{\star \star} P<0.005,{ }^{\star \star \star} P<0.001,{ }^{\star \star \star \star} P<0.0001\right)$. At 30 d.p.i., mice receiving cotransfer of WT and $1 / 2^{-} /-$donor cells were infected with heterosubtypic virus (X31-OVA ${ }_{\|}$). (c,d) Representative histograms of forward scatter (FCS) and CD69 expression on donor populations on day 2 post-X31-OVA $\mid$ challenge. Grey histograms represent donor staining at 30 d.p.i. before heterosubtypic challenge. (e) Number of donor cells in lungs of primed mice before and 4 days post-heterosubtypic challenge ( $n=4$ mice per group per time point; one of two experiments). Significant differences determined with unpaired, two-tailed, Student's $t$-tests $\left(\alpha=0.05\right.$ and ${ }^{\star} P<0.05, \star \star P<0.005$, $\left.{ }^{\star \star \star} P<0.001,{ }^{\star \star \star \star} P<0.0001\right)$. All error bars represent the s.d.

which at high doses should mimic autocrine IL-2. We injected IL2C from 5-7 d.p.i., corresponding to the timeframe when MHCII interactions were required for optimum memory formation (Fig. 1). The IL-2C effectively signalled $I 2^{-/-}$effector cells as indicated by increased ex vivo STAT5 but not STAT3 phosphorylation at 7 d.p.i. (Fig. 4a), and enhanced CD25 and CD122 expression comparable to levels on WT donors (Fig. 4b).

Strikingly, IL-2C treatment restored $\mathrm{Il} 2^{-/-}$memory at 28 d.p.i. to WT levels but did not impact the peak expansion of $\mathrm{Il}^{-/}-$cells at 7 d.p.i. (Fig. 4c). The effect of IL-2C treatment was long-lived as $I l 2^{-/}-$donor cell recovery in IL-2C-treated mice was still equivalent to WT donor populations at over 100 d.p.i. (Fig. $4 \mathrm{~d}$ ). Optimal rescue required a high dose of $2 \mu \mathrm{g}$ of IL2 , while $1 \mu \mathrm{g}$ led to reduced but still improved $I l 2^{-/-}$donor cell survival (Fig. 4e). Late IL-2C treatment also enhanced the survival of WT donor cells (Fig. 4f), suggesting that IL-2 production by effectors is normally limiting for optimum memory generation.

In contrast to its ability to restore survival of primed donor cells, IL-2C given at 5-7 d.p.i. did not enhance recovery of naive $I l 2^{-/-}$donor cells at 28 d.p.i. (Fig. $4 \mathrm{~g}$ ). As naive cells may require early IL-2 signals to 'programme' the capacity to form memory ${ }^{9}$, we treated recipients of naive $I l 2^{-/-}$donors at $1-3$ d.p.i. or at both 1-3 and 5-7 d.p.i. with IL-2C. Only treatment at both 1-3 and 5-7 d.p.i. restored memory generation (Fig. 4g). We conclude that naive CD4 $\mathrm{T}$ cells require both early IL-2dependent programming and later IL-2 signals to efficiently become memory following IAV challenge. In contrast, IL-2primed cells no longer require early IL-2 programming but are as dependent on late IL-2 at the effector stage for effective memory generation.

Late IL-2-induced CD127 on effectors correlates with memory. To search for late IL-2-dependent changes that might lead to better memory generation, we compared gene expression from WT and $I l 2^{-}-$effectors at 7 d.p.i. by microarray. We found only 88 genes differentially expressed at a threshold of $>1.5$ and $P<0.05$ (Supplementary Data set I). DAVID functional annotation clustering revealed five pathways with $P$-values $<0.05$ and $>1.3$-fold enrichment (Fig. 5a). $I l 7 r$, encoding the IL-7 receptor $\alpha$ chain (CD127), was decreased in $I l 2^{-/-}$effectors and was common to three of the five pathways (Fig. 5b). When a more conservative $\geq 2$-fold threshold was applied, only 27 genes, including $I l 7 r$, were differentially expressed by the WT and $I l 2^{-/-}$effector cells (Fig. 5c). As IL-7 is a critical and limiting factor regulating T-cell homeostasis and memory T-cell survival ${ }^{24-26}$, decreased CD127 expression could be a major factor limiting the ability of $I l 2^{-/-}$effector cells to successfully transition to memory.

To directly evaluate the impact of late IL-2 on effector cell CD127 expression, we analysed donor cells at 7 d.p.i. by flow cytometry. Il2 ${ }^{-/-}$effectors expressed lower CD127 than WT cells, and IL-2C treatment at 5-7 d.p.i. restored CD127 on $\mathrm{Il}^{-/}-$cells to WT levels (Fig. 5d). Importantly, enhanced CD127 expression by $I l 2^{-/-}$cells following IL-2C treatment at 5-7 d.p.i. was maintained at 28 d.p.i. (Fig. 5d), and the level of CD127 expression was proportional to the dose of IL-2 administered (Fig. 5e, f). Furthermore, treatment of recipients of WT DO11.10 donor cells with IL-2 neutralizing Ab reduced their CD127 expression at 7 and 28 d.p.i. (Fig. 5g). IL-2 neutralization during the first week of IAV infection also reduced CD127 expression on polyclonal donor CD4 T cells present at 28 d.p.i. (Fig. 5h). Finally, IL-2C treatment of recipients of naive $I l 2^{-/}-$donor cells at both $1-3$ and 5-7 d.p.i., but not at either time point alone, induced enhanced CD127 expression (Fig. 5i). These results indicate that the level of CD127 expression on both primary and secondary effector CD4 $\mathrm{T}$ cells is directly linked to IL-2 exposure at 5-7 d.p.i. and closely correlates with memory cell recovery. 

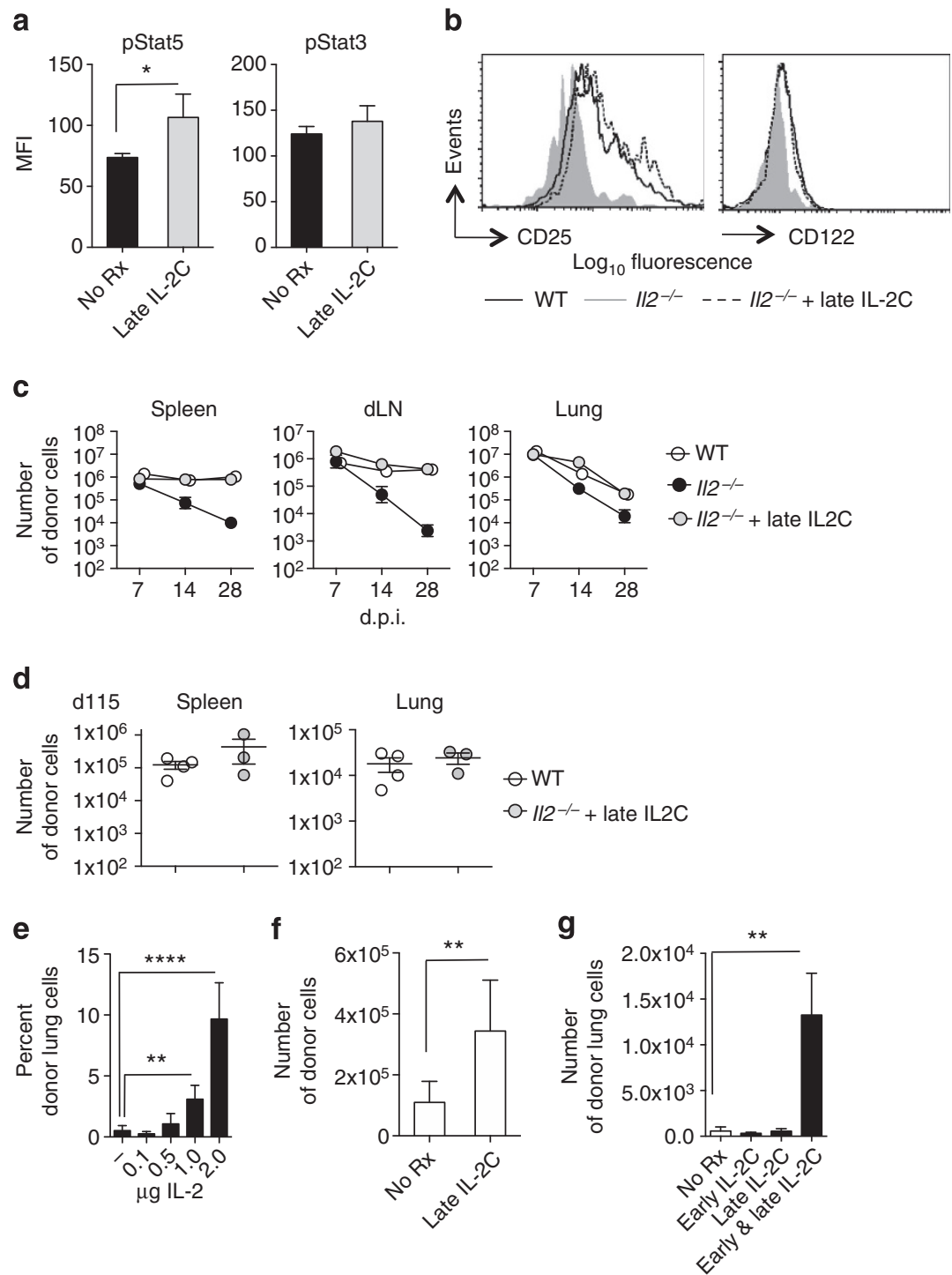

Figure 4 | A late IL-2 dependent checkpoint controls memory CD4 T-cell survival. Unprimed BALB/c mice receiving primed WT or II2-/- donor cells were challenged with IAV and treated with IL-2C on 5-7 d.p.i. (late IL-2C). (a) pSTAT5 and pSTAT3 mean fluorescent intensity (MFI) of II2 ${ }^{-/-}$donor cells ex vivo and (b) CD25 and CD122 expression on donor cells, with and without late IL-2C at 7 d.p.i. (one of three experiments). Significant differences determined with unpaired, two-tailed, Student's t-tests $\left(\alpha=0.05\right.$ and $\left.{ }^{\star} P<0.05\right)$. (c) Longitudinal analysis of recipients of WT or $I 12^{-/}-{ }_{\text {donors }}$ with and without late IL-2C (four mice per day per group; one of two experiments). (d) Recovery of donor cells 115 d.p.i. (one of two experiments). (e) Recipients of $112^{-/-}$cells were treated from 5 to 7 d.p.i. with IL-2C containing stated amounts of IL-2 and donor cells detected in the lung at 28 d.p.i. (four mice per group). Significant differences in one of two replicate experiments were determined following one-way ANOVA analysis with Bonferroni's multiple comparison post-test ${ }^{\star \star} P<0.005$, $\left.{ }^{\star \star \star \star} P<0.0001\right)$. (f) Recovery of WT donor cells from hosts treated with or without late IL-2C (1 of 2 experiments). Significant differences were determined with unpaired, two-tailed, Student's $t$-tests $\left(\alpha=0.05\right.$, and $\left({ }^{\star \star} P<0.005\right)$. (g) Recipients of naive $112-/-$ cells were treated on stated d.p.i. with IL-2C and donor cells detected in the lung at 28 d.p.i. (four mice per group; (one of three experiments)). Significant differences determined following one-way ANOVA analysis with Bonferroni's multiple comparison post-test $(\star \star P<0.005)$. All error bars represent the s.d.

Enhanced access to IL-7 rescues survival of $\mathrm{Il} 2^{-/-}$memory. We treated recipients of $I l 2^{-/-}$donors with IL-7: anti-IL-7 complexes (IL-7C) ${ }^{27}$ to determine whether enhanced IL-7 signals could overcome the need for late IL-2 to facilitate memory generation. IL-7C treatment from 5 to 7 d.p.i. failed to rescue donor cell survival (not shown). However, when administered every other day starting at 14 d.p.i. (following viral clearance ${ }^{4}$ ) through 26 d.p.i., IL-7C treatment restored $I 2^{-/-}$donor cell recovery to levels comparable to those achieved by IL-2C treatment from 5 to 7 d.p.i. (Fig. 6a). These results suggest that late IL-2 induces permanent upregulation of CD127 on effector cells, thus increasing their ability to compete for ambient IL-7 that is required for their long-term persistence.
In nude mice, access to IL-7 is greater because it is not limited by competition from host $\mathrm{T}$ cells, but survival of transferred CD4 $\mathrm{T}$ cells is still IL-7-dependent ${ }^{26}$. Therefore, we predicted that in nude hosts the $I l 2^{-1-}$ donor cells would have greater access to IL7 and would perform more like WT donor cells. Indeed, we found equivalent long-term survival of WT and $I 2^{-/-}$donor cells in IAV-challenged nude hosts (Fig. 6b), suggesting that when ambient IL-7 levels are higher, a substantial level of memory generation is seen without late IL-2 production by effector CD4 T cells.

Late IL-2 limits acute apoptosis of effector cells. To analyse whether additional IL-2-dependent mechanisms improve the 


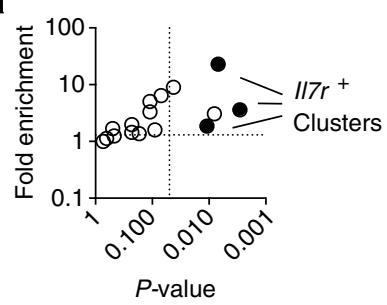

b

\begin{tabular}{l|c|l}
$\begin{array}{l}\text { Cluster: Gene } \\
\text { ontogeny pathway }\end{array}$ & $P$-value & Genes \\
\hline $\begin{array}{l}\text { 1: Regulation of } \\
\text { lymphocyte-mediated } \\
\text { immunity }\end{array}$ & 0.006 & II7r, H2-q1, KIre1 \\
\hline $\begin{array}{l}\text { 2: Protein-tyrosine } \\
\text { phosphatase }\end{array}$ & 0.042 & Cdc14b, Ptpn13, Ptprk \\
\hline 3: Homeostatic process & 0.002 & $\begin{array}{l}\text { Il7r, Cd7, Mt1, Mt2, Ckb, } \\
\text { SIc121a, Hba-1 }\end{array}$ \\
\hline $\begin{array}{l}\text { 4: Phosphorus } \\
\text { metabolic process }\end{array}$ & 0.008 & $\begin{array}{l}\text { Riok3, Wwox, Ddr1, } \\
\text { Ephx4, Fes, Gm3555, } \\
\text { Pim1, Tpi1, Vrk1 }\end{array}$ \\
\hline 5: Plasma membrane & 0.011 & $\begin{array}{l}\text { Il7r, CD38, Cd7, Gpr160, } \\
\text { Cysltr1, Cysltr2, Ddr1, } \\
\text { Icam2, Ifngr1, KIra3, } \\
\text { Ltb4r1, Ptprk, Sectm1a, } \\
\text { Treml2, Slc2a1 }\end{array}$
\end{tabular}

C

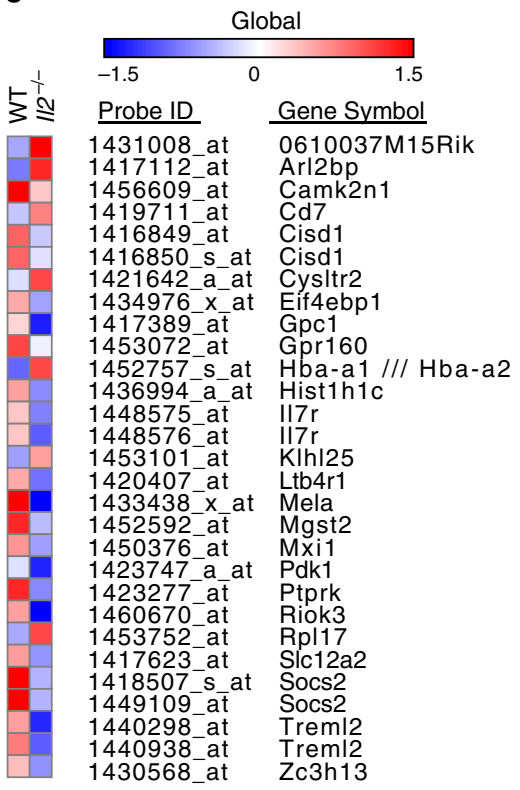

d
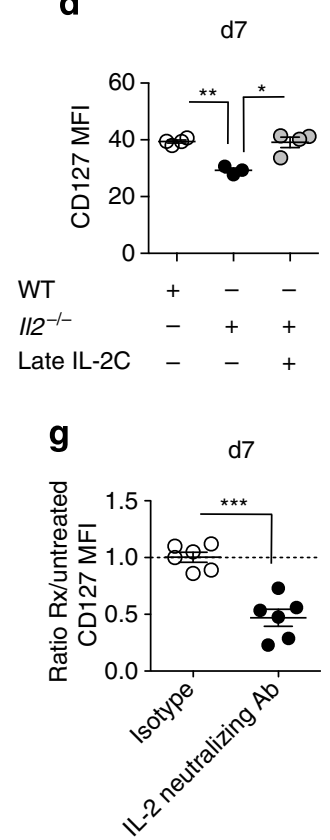

d28
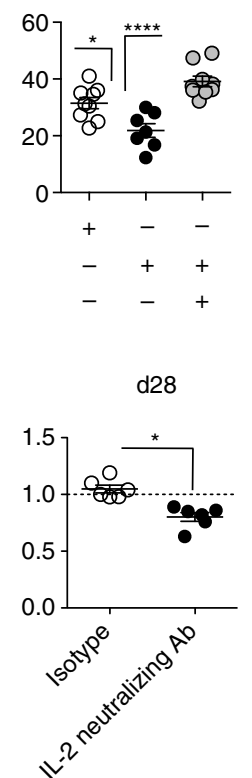

e

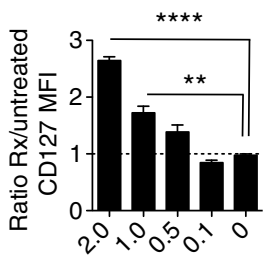

$\mu \mathrm{g} \mathrm{IL-2}$ f

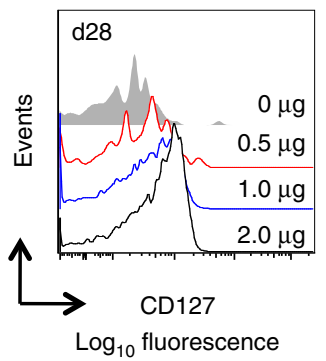

i

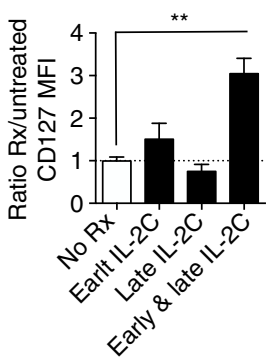

Figure 5 | IL-2-dependent CD127 induction at the effector phase is associated with memory generation. Primed WT or $112^{-/-}$DO11.10 cells were sort purified from the lungs of IAV-challenged recipients at 7 d.p.i. in two replicate experiments. (a) Genes differentially expressed by microarray segregated into 16 clusters by David functional annotation analysis. The fold enrichment and $P$-value of the most significant pathways in each cluster are shown ( $>1.3$ and $<0.05$, respectively). (b) The gene ontogeny pathway and enriched genes within clusters of interest represented in (a). (c) Summary of differentially expressed genes between WT and $112^{-/-}$donors with a threshold of $\geq 2$-fold and $P<0.05$. (d) Surface expression of CD127 at 7 and 28 d.p.i. on WT and $112^{-/-}$donors from individual mice treated as indicated (one of three experiments). (e,f) Summary and representative CD127 staining on $112^{-/-}$donors at 28 d.p.i. from mice treated with stated amounts of IL-2C from 5 to 7 d.p.i., summary from five mice/group (one of two experiments). (g) Ratio of CD127 expression on WT donor cells in the lung at 7 and 28 d.p.i. from mice either treated with IL-2 neutralizing or control Ab on 1-7 d.p.i. (1 of 2 experiments). (h) Ratio of CD127 expression on polyclonal donor CD4 T cells from IAV-primed C57BL/6 mice on 28 d.p.i. from host mice either treated with IL-2 neutralizing or control Ab on 1-7 d.p.i. (one of two experiments). (i) Ratio of CD127 expression on naive $112^{-/-}$donors treated on stated d.p.i. with IL-2C (four mice per group; one of three experiments). Significant differences were determined on raw data following one-way ANOVA analysis with Bonferroni's multiple comparison post-test $(\mathbf{d}, \mathbf{e}, \mathbf{i})$ or determined with unpaired, two-tailed, Student's $t$-tests $(\mathbf{g}, \mathbf{h}$, with $\alpha=0.05$ and $\left.{ }^{\star} P<0.05,{ }^{\star \star} P<0.005,{ }^{\star \star \star} P<0.001,{ }^{\star \star \star \star} P<00.0001\right)$. All error bars represent the s.d. 

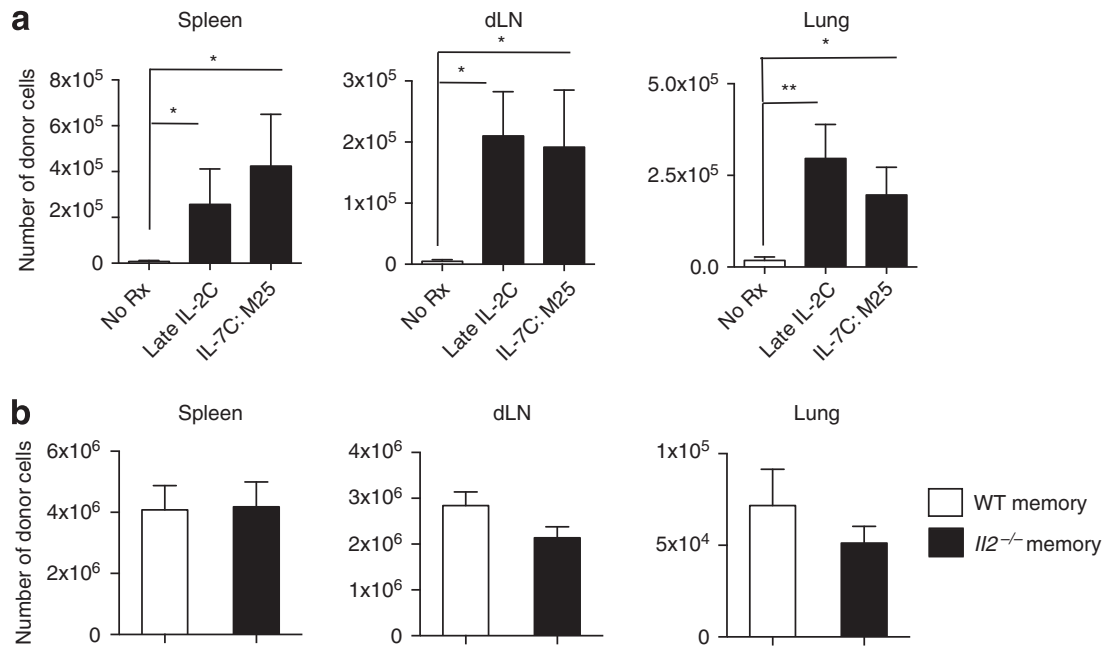

C

dLN
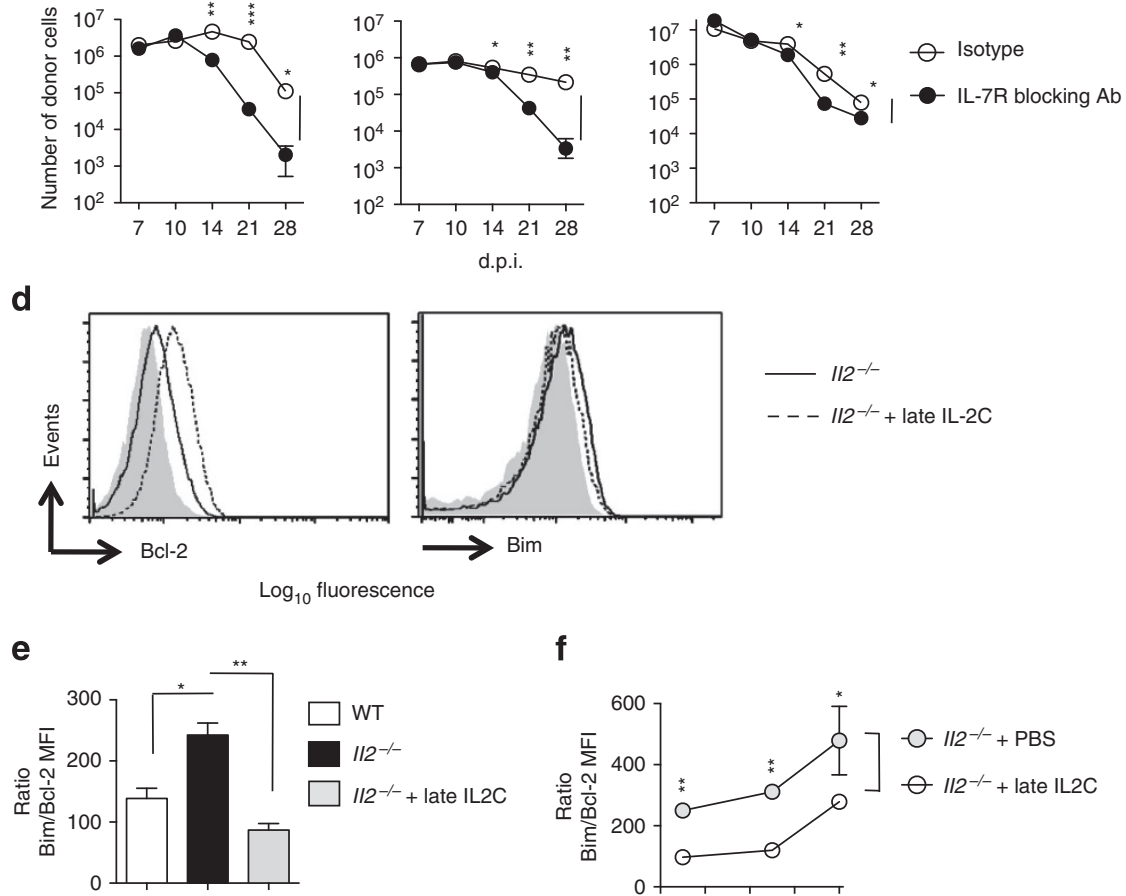

$---112^{-/-}+$late IL-2C

f

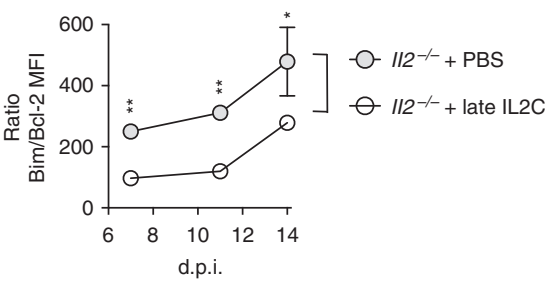

Figure 6 | IL-2-mediated rescue limits acute apoptosis of effector cells. WT recipients of primed II2-/- DO11.10 cells challenged with IAV were treated with IL-2C from 5 to 7 d.p.i. or with IL-7C (using anti-IL-7 Ab clone M25) every other day from 14 to 26 d.p.i. (a) The number of donors at 28 d.p.i. is shown (five mice per group; one of two experiments). (b) The number of WT or $112^{-/-}$donors at 28 d.p.i. in nude recipients challenged with IAV (five mice per group; one of three experiments). WT recipients of primed WT DO11.10 cells challenged with IAV were treated with isotype control or IL-7 receptor blocking Ab every other day from 7 to 26 d.p.i. (c) Number of donors detected at the stated d.p.i. (four mice per group; one of two experiments). WT recipients of primed $I 12^{-/-}$DO11.10 cells were infected with IAV and were untreated or treated with late IL-2C. (d) Representative donor cell Bcl-2 (left) and Bim (right) expression at 7 d.p.i., from four mice per group. Isotype staining is shown as a grey histogram. (e) The Bim/Bcl-2 MFI ratio was determined for groups described in (d) versus WT donors (four mice/group; one of two separate experiments). (f) Kinetics of Bim/Bcl-2 MFI ratio in $112^{-/-}$donors treated as described on stated days following IAV infection (four mice per group; one of two separate experiments). Significant differences were determined following one-way ANOVA analysis with Bonferroni's multiple comparison post-test (a,e) or determined with unpaired, two-tailed, Student's $t$-tests (c and $\mathbf{f}$, with $\alpha=0.05$ and ${ }^{\star} P<0.05,{ }^{\star \star} P<0.005,{ }^{\star \star \star} P<<0.001$, ${ }^{\star \star \star \star} P<0.0001$ ) on raw data. All error bars represent the s.d.

capacity of effector cells to form memory, or whether enhanced fitness to access to IL-7 is the dominant factor, we treated recipients of WT donor cells with blocking Ab against CD127 every other day from 7 to 28 d.p.i. to disrupt IL-7 signalling ${ }^{28}$. Blocking IL-7 access substantially reduced donor cell recovery at 28 d.p.i. (Fig. 6c), confirming that sustained access to IL-7 is necessary for optimal memory survival. However, CD127 blockade did not reduce donor recovery at 14 d.p.i., when the effect of IL-2deficiency was already striking (see Fig. 2), and the reduction in donor cell recovery at 28 d.p.i. was not as marked as that seen following IL-2 neutralization. Thus, autocrine IL-2 signals during 5-7 d.p.i. must block acute apoptosis of effector cells before 14 
d.p.i. by an IL-7-independent pro-survival mechanism ${ }^{13}$, resulting in a larger pool of effectors able to compete for survival as memory.

We analysed expression of the pro-apoptotic molecule Bim versus the anti-apoptotic molecule Bcl-2, which plays key opposing roles in regulating T-cell lifespan ${ }^{29}$, from 7 to 14 d.p.i. to ask whether late IL-2 signals impacted their expression by effector CD4 $\mathrm{T}$ cells responding against IAV. The Bim/Bcl-2 ratio was higher in $I l 2^{-/-}$than in WT donors at 7 d.p.i. and when the in situ survival of $I l 2^{-/}$effectors was enhanced by IL-2C treatment the $\mathrm{Bim} / \mathrm{Bcl}-2$ ratio was reduced (Fig. 6d,e) from 7 through 14 d.p.i. (Fig. 6f). $I l 2^{-/-}$effectors also expressed higher levels of TNF-related apoptosis inducing ligand (TRAIL), which can potentiate effector CD4 T-cell apoptosis ${ }^{30}$ (Supplementary Fig 4). These findings suggest that late IL-2 signals also enhance the efficiency of memory generation by directly limiting acute apoptosis of effector cells.

Late CD27:CD70 signals enhance memory through IL-2 induction. CD27:CD70 interactions enhance IL-2 production by and CD127 expression on CD8 $\mathrm{T}$ cells ${ }^{31-34}$, and IL-2 production by $\mathrm{CD} 4 \mathrm{~T}$ cells ${ }^{35}$. To determine a connection between late cognate interactions between effectors with APC and resulting autocrine IL-2 induction, we asked whether CD27:CD70 interactions enhanced memory CD4 T-cell generation following IAV challenge. We analysed expression of CD27 on WT CD4 $\mathrm{T}$ cells and $\mathrm{CD} 70$ on $\mathrm{CD} 1 \mathrm{c}^{+}$cells to determine whether a potential interaction was plausible during the late memory checkpoint identified here. Donor CD27 expression fell sharply during 5-7 d.p.i., while a reciprocal increase in $\mathrm{CD}^{+}{ }^{+} \mathrm{CD} 11 \mathrm{c}^{+}$ cells was observed (Fig. 7a), in agreement with previous analysis ${ }^{36}$, and confirming the potential for CD27:CD70 signalling during 4-7 d.p.i. We could not detect CD70 on T cells (not shown).

We then treated recipients of primed WT donor cells with anti$\mathrm{CD} 70$ or isotype control $\mathrm{Ab}$ from 1 to 6 d.p.i. and found enhanced donor CD27 expression at 7 d.p.i. after CD70 blockade (Fig. 7b,c), suggesting that the donor cells do interact with CD70 expressed by APC during the memory checkpoint ${ }^{37}$. Anti-CD70 Ab treatment did not impact viral titres detected at 7 d.p.i. (Fig. 7d) nor did treatment have an impact on the peak expansion of donor cells (Fig. 7e). Strikingly, however, anti-CD70 Ab a

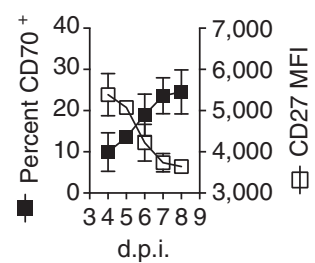

d.p.i. b

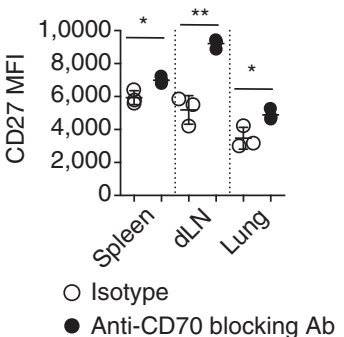

C

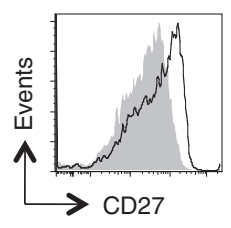

Isotype

Anti-CD70 blocking Ab

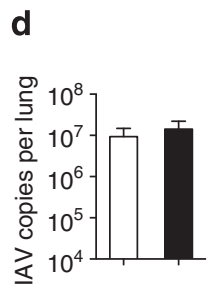

e

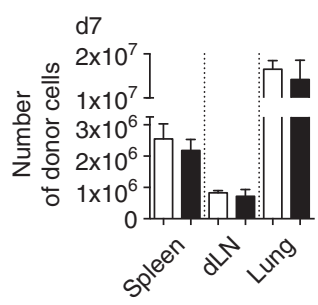

$2 e^{1 e^{e r}} d v^{2} v^{2 n s}$

f

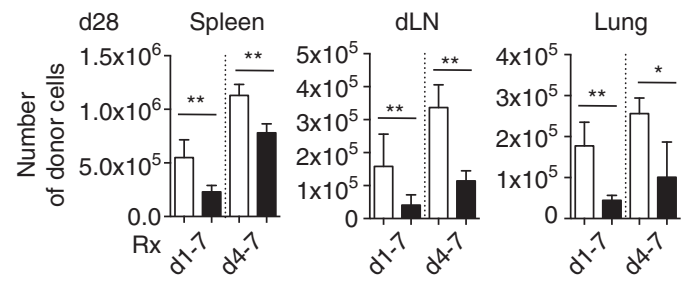

$\square$ Isotype

Anti-CD70 blocking Ab g

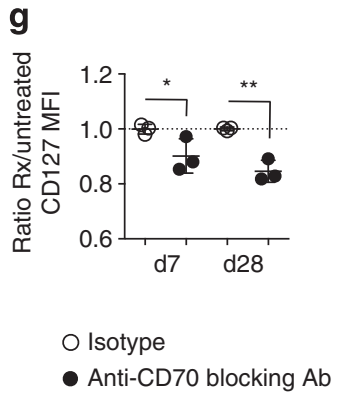

h
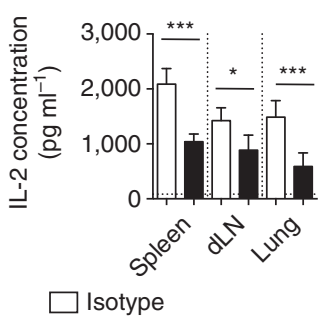

Anti-CD70 blocking Ab i

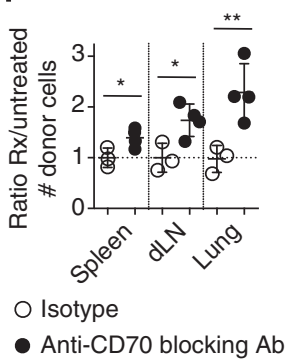

Figure 7 | Late CD27:CD70 signals contribute to memory CD4 T-cell survival by enhancing IL-2 production. Unprimed BALB/C mice receiving primed WT D011.10 donor cells were challenged with IAV. (a) Expression of CD27 on donor cells (MFI) and the $\% \mathrm{CD}^{\circ} \mathrm{C}^{+} \mathrm{CD} 11 \mathrm{c}^{+} \mathrm{MHC}-\mathrm{II}^{+} \mathrm{APC}$ in the $\mathrm{dLN}$ on the stated d.p.i. (three mice per group; one of three experiments). Recipients of WT donor cells challenged with IAV were treated with isotype or anti-CD70 blocking Ab on 1-7 d.p.i. (b) Donor cell CD27 MFI, (c) representative CD27 surface expression, (d) viral titre and (e) donor cell recovery at 7 d.p.i. with and without blockade on 1-7 d.p.i. (f) Donor cell recovery on 28 d.p.i. with CD70 blockade on the indicated days. (g) Ratio of CD127 expression on donor cells on $\mathrm{d} 7$ and $\mathrm{d} 28$ in isotype and anti-CD70 blocking Ab-treated animals (three mice per group; one of three experiments). (h) IL-2 production of donor cells isolated at 7 d.p.i. from isotype and anti-CD70 blocking Ab-treated animals stimulated ex vivo with peptide for $18 \mathrm{~h}$ ( 1 of 2 experiments). (i) Recovery of donor cells in isotype and anti-CD70 blocking Ab-treated animals that were also treated with late IL-2C (four mice per group; one of two experiments). All error bars represent the s.d. and significant differences determined with unpaired, two-tailed, Student's $t$-tests ( $\alpha=0.05$ and $\left.{ }^{\star} P<0.05,{ }^{\star \star} P<0.005,{ }^{\star \star \star} P<0.001\right)$ on raw data. 
treatment significantly reduced donor cell recovery at 28 d.p.i. (Fig. 7f) and treatment reduced CD127 expression on surviving cells (Fig. 7g). Importantly, when we restricted anti-CD70 Ab treatment to 4-7 d.p.i., donor cell survival was comparably reduced to that observed with treatment from 1 to 7 d.p.i. (Fig. 7f). This implies that the key CD70-dependent signals regulating memory $\mathrm{CD} 4 \mathrm{~T}$-cell generation occur late during the effector phase instead of during initial interactions with APC.

As expected ${ }^{35}$, anti-CD70 Ab treatment reduced effector cell IL-2 production (Fig. 7h). When we treated recipients of WT donor cells with both anti-CD70 Ab from 4 to 7 d.p.i. and with IL-2C from 5 to 7 d.p.i., donor cell survival at 28 d.p.i. was restored to that observed in isotype Ab-treated mice (Fig. 7i), implying that CD70 blockade impaired memory generation primarily by reducing late IL-2 production by effector cells. Donor recovery was actually slightly enhanced in mice receiving IL-2C and anti-CD70 Ab versus IL-2C and control Ab. This finding may reflect that CD70 delivers pro-apoptotic signals to some of the effector cells ${ }^{38}$. We conclude that late CD27:CD70 signals improve memory generation by enhancing IL-2 production by effector CD4 $\mathrm{T}$ cells during interactions with APC. This is further evidence that key fate decisions governing CD4 T-cell survival leading to memory are made at the effector phase during late, presumably cognate, interactions with APC.

\section{Discussion}

Our studies identify a novel and discrete checkpoint during the CD4 T-cell response against IAV that determines the generation of long-lived memory. Efficient transition to memory requires both MHC-II and CD27:CD70 interactions between 4 and 7 d.p.i., implying that the effectors must engage in a late cognate interaction, and we find that $\mathrm{CD} 11 \mathrm{c}^{+} \mathrm{APC}$ are sufficient for this implied interaction. We show that the majority of effectors responding against the virus express a default programme for death unless this interaction with APC causes them to produce high levels of IL-2. Autocrine IL-2, or high levels of exogenous IL2 delivered in the form of IL-2C, directly rescues some effectors from acute apoptotic death, and also acts in a dose-dependent manner to upregulate CD127 expression. Once upregulated by IL-2, higher expression of CD127 is maintained and increases the capacity of cells to compete for ambient IL-7 that sustains their long-term persistence.

The fact that IL-2 production by $\mathrm{CD} 4 \mathrm{~T}$ cells requires TcR stimulation $^{39}$, and that both late CD70 and MHC-II expression are required for optimal long-term survival of effector CD4 T cells following IAV challenge, strongly supports the concept that the effector CD4 T cell: APC interaction is the first step in the memory checkpoint. Expressions of CD70 and MHC-II are tightly linked ${ }^{40}$, and levels of CD70 on APC and of CD27 on T cells are quickly downregulated following their interaction, resulting in a potent negative feedback loop ${ }^{37}$. This and the rapid kinetics of default apoptosis of effector CD4 T cells likely help define the timeframe during which late cognate interaction must occur to rescue a cohort of cells to survive and transition to memory. Other signals could also be involved as we note that CD70 blockade did not have as marked impact on reducing memory generation as did blockade of IL- 2 .

We found similar kinetics of effector contraction in the lung, spleen and dLN, consistent with uniform T-cell effector apoptosis in diverse organs following peptide immunization ${ }^{41}$. Effectors responding against IAV in different organs are marked by wide differences in phenotype, cytokine profile and gene expression $^{14,42}$, and thus the late IL-2-dependent memory checkpoint defined here appears to act broadly rather than in a subset-specific manner. We did, however, consistently identify a population of long-lived IL-2-independent memory cells in the lung. Unique mechanisms may govern the generation and persistence of tissue-resident memory CD4 T cells in the lung, as has been reported with brain-resident memory CD8 T cells ${ }^{43}$.

Infection with live IAV results in high levels of Ag presentation and inflammation throughout the period of CD4 T-cell priming and expansion, followed by rapid viral clearance. This may be required to drive effectors to the state in which they die by default unless they re-encounter APC, and make and bind IL-2. Nonreplicating, shorter-term Ag exposure may result in less differentiated effectors ${ }^{44}$, and it remains to be determined if such effectors have the same requirements for the memory checkpoint identified here. Studies describing 'stem cell memory ${ }^{45}$ suggest that some less-differentiated effectors that may not yet have become programmed to undergo default apoptosis can efficiently enter the memory pool. However, we point out that during vigorous responses against pathogens the number of effector $\mathrm{T}$ cells at the peak of response far exceed those at earlier time points, and so such effectors would appear to have a greater potential to dominate the memory pool. Furthermore, more differentiated effector populations are comprised of many functionally specialized subsets. For example, IAV infection generates $\mathrm{T}_{\mathrm{FH}}$ in secondary lymphoid organs and cytotoxic CD4 $\mathrm{T}$ cells in the lung by 7-8 d.p.i. ${ }^{46}$ As virtually no memory cells develop in secondary lymphoid organs without late IL-2 signals, even though there are less divided donors with central memory phenotypes in these organs 42 , it appears that in this model effector cells at various states of differentiation require late IL-2 to generate memory.

Memory CD4 $\mathrm{T}$ cells provide broad spectrum immunity and robust protection against $\mathrm{IAV}^{4,47,48}$ and other viral infections ${ }^{47}$ and are not as readily evaded by new variants that rapidly mutate to avoid neutralizing Abs. Thus, the case for developing vaccines that induce strong $\mathrm{CD}^{+}{ }^{+}$as well as $\mathrm{CD} 8{ }^{+} \mathrm{T}$ cell and long-term $\mathrm{B}$-cell memory, including $\mathrm{Ab}$, is compelling. Our results suggest a new paradigm for when and how CD4 T-cell memory formation is regulated following infection. Effective cognate interaction at the effector phase is necessary to induce optimal autocrine IL-2 signalling that is required to limit apoptosis of effectors and to sustain their transition to and persistence as memory cells. Moreover, boosting CD4 T-cell memory is equally dependent on this late memory checkpoint. Thus, we predict that vaccines that provide both Ag and adjuvant effects that span the CD4 T-cell effector phase and hence better mirror natural infection will be better able to induce robust memory that includes multiple functional subsets ${ }^{47}$.

\section{Methods}

Mice. Naive $\mathrm{CD}_{4}{ }^{+} \mathrm{T}$ cells were obtained from 5- to 8-week-old male or female DO11.10.Thy1.2, DO11.10.Thy1.2/Thy1.1 and Il2 ${ }^{-/-}$DO11.10.Thy1.2 mice originally provided by A. Abbas (UCSF). Recipients of cell transfers were male BALB/ c.Thy1.2 or BALB/c.Thy1.1 or nude mice that were at least 8 weeks old. OTII.Thy1.1, CD11c Tg.H2-Ab1 ${ }^{-/-}$and C57Bl/6 Thy1.1 or Thy1.2 mice were used in certain experiments. T-cell-deficient nude mice were purchased from Charles River Laboratories. All other mice were obtained from the breeding facility at Trudeau Institute, the University of Massachusetts Medical School, or Jackson Laboratories. The Animal Care and Use Committee at Trudeau Institute (Saranac Lake, NY) or the Institutional Animal Care and Use Committee of the University of Massachusetts Medical School (Worcester, MA) approved all experimental animal procedures.

CD4 T-cell isolation and in vitro-primed memory generation. Naïve CD4 ${ }^{+}$ $\mathrm{T}$ cells were obtained from pooled spleen and peripheral lymph nodes as previously described ${ }^{42}$. In brief, cells were purified through nylon wool and percoll gradient separation. CD4 T cells were isolated by positive CD4 MACS selection (Miltenyi). Resulting $\mathrm{CD} 4{ }^{+}$cells routinely expressed a characteristic naive phenotype (small size, CD62L $\mathrm{L}^{\text {hi }}, \mathrm{CD} 44^{\text {lo }}$ and $\mathrm{CD} 25^{\mathrm{lo}}$ ) $>97 \% \mathrm{TcR}^{+} . \mathrm{T}_{\mathrm{H}} 1$-polarized effectors were generated in vitro as described ${ }^{49}$. In brief, naive WT or Il2 ${ }^{-/-} \mathrm{CD} 4 \mathrm{~T}$ cells were cultured with an equal number of irradiated APC $\left(2 \times 10^{5}\right.$ per $\left.\mathrm{ml}\right)$ in the presence 
of exogenous IL-2 (20 $\mathrm{ng} \mathrm{ml}^{-1}$ ), $2 \mathrm{ng} \mathrm{ml}^{-1} \mathrm{IL}-12$ (Peprotech), $10 \mathrm{\mu g} \mathrm{ml}^{-1}$ anti-IL$4 \mathrm{Ab}$ (11B11; Bioxcell) and $5 \mu \mathrm{M} \mathrm{OVA}_{\mathrm{II}}$ peptide. In vitro-primed memory cells were obtained by thoroughly washing effector cultures at 4 days and re-culturing the cells in fresh media for at least 3 days in the absence of $\mathrm{Ag}$ and exogenous cytokines. Live cells were isolated by lymphocyte separation (Cedarlane). In some experiments, total polyclonal CD4 T cells were isolated from IAV-primed C57BL/ 6.Thy1.1 mice by positive selection with CD4 MACS beads (Miltenyi), and CFSE labelled as previously described ${ }^{50}$. A total of $1 \times 10^{6} \mathrm{TcR} \mathrm{Tg}$ donor cells (naive or memory) were adoptively transferred in all experiments, a number found previously to be required to reliably detect donor cells following IAV challenge in the memory phase ${ }^{4}$. Alternatively, $1 \times 10^{7}$ polyclonal memory cells were transferred to adoptive hosts, as in previous studies ${ }^{14}$. All donor CD4 T cells were adoptively transferred in $200 \mu \mathrm{l}$ PBS by intravenous (i.v.) injection.

Virus stocks and infections. Influenza A/PR8-OVA ${ }_{\mathrm{II}}(\mathrm{H} 1 \mathrm{~N} 1)$ and $\mathrm{A} / \mathrm{HKx} 31$ $\mathrm{OVA}_{\mathrm{II}}(\mathrm{H} 3 \mathrm{~N} 2)$ viruses were produced in the allantoic cavity of embryonated hen eggs from stock obtained from P. Doherty. Mice were infected intranasally under light isoflurane anaesthesia (Webster Veterinary Supply) with a sublethal $0.2 \mathrm{LD}_{50}$ dose of A/PR8-OVA ${ }_{\text {II }}$ virus or 25 Hemagglutination Units of A/HKx31-OVA $A_{I I}$ in $50 \mu \mathrm{l}$ PBS. Donor cell injection and viral infection occurred on the same day.

Detection of IAV titre. Pulmonary viral titre was determined by quantitation of viral RNA. RNA was prepared from whole-lung homogenates using TRIzol (Sigma-Aldrich), and $2.5 \mu \mathrm{g}$ of RNA was reverse transcribed into cDNA using random hexamer primers and Superscript II Reverse Transcriptase (Invitrogen). Quantitative PCR was performed to amplify the acidic polymerase (PA) gene of A/PR8-OVA ${ }_{\text {II }}$ using an ABI Prism 7700 Sequence Detector (Applied Biosystems) with $50 \mathrm{ng}$ of cDNA per reaction and the following primers and probe: forward primer, 5'-CGGTCCAAATTCCTGCTGA-3'; reverse primer, 5'-CATTGGGTTCC TTCCATCCA-3'; probe, 5'-6-FAM-CCAAGTCATGAAGGAGAGGGAATACC GCT- ${ }^{\prime}$. Data were analyzed with Sequence Detector v1.7a (Applied Biosystems). The copy number of the PA gene per 50 ng of cDNA was calculated using a PA-containing plasmid of known concentration as a standard. The number of copies of PA gene per lung is presented.

Cytokine complex and neutralizing Ab treatments. For some experiments, mice were treated on indicated days with injections of cytokine: anti-cytokine monoclonal $\mathrm{Ab}$ complexes. For IL-2 complexes (IL-2C), mice received $2 \mu \mathrm{g}$ per day of recombinant IL-2 (eBioscience) premixed with $20 \mu \mathrm{g}$ of anti-mouse IL-2 monoclonal (m) Ab clone S4B6-1 (S4B6) (BD Pharmingen). In certain experiments, the amount of IL-2 complexes was varied, as indicated. For IL-7 complexes, (IL-7C), mice received $1.5 \mu \mathrm{g}$ of recombinant mouse IL-7 (eBioscience) premixed with $15 \mu \mathrm{g}$ of anti-IL-7 mAb clone M25 (BioXcell). Complexes were incubated at room temperature for 20 min before intraperitoneal (i.p.) injection in $200 \mu \mathrm{l}$ of PBS.

In other experiments mice were treated as indicated with $0.25 \mathrm{mg}$ per day of both anti-IL-2 Abs S4B6 and JES6-1A12 (Rat IgG2a), $0.5 \mathrm{mg}$ per day of anti-IL-7 receptor blocking $\mathrm{Ab}$ (A7R34; Rat IgG2a), $0.5 \mathrm{mg}$ per day of anti-CD70 Ab (FR70), $1 \mathrm{mg}$ per day of anti-MHC-II (M5/114) or appropriate isotype control Ab (all BioXcell) on stated days. Ab was delivered by i.p. injection in $200 \mu \mathrm{l}$ of PBS, with the exception of anti-MHC-II Ab that was delivered i.v.

Flow cytometry and intracellular cytokine staining. The lung, dLN and spleen were prepared into single cell suspensions by mechanical disruption and passage through a nylon membrane. Flow cytometry was performed as described ${ }^{42}$, using fluorochrome-labelled Abs at manufacturer's recommended 1:50 to 1:100 dilutions for surface staining including anti-Thy1.1 (OX-7), anti-Thy1.2 (53-2.1), anti-CD4 (RM4.5), anti-CD62L (MEL14), anti-CD69 (H12F3), anti-CD44 (1M7), anti-CD27 (LG.3A10), anti-CD25 (PC61), anti-CD127 (IL-7R, A7R34), anti-CD278 (ICOS, 7E.17G9), anti-CD134 (OX40, OX-86), anti-CD70 (FR70), anti-CD253 (TRAIL, N2B2) and anti-MHC-II (M5/114) (Pharmingen, eBioscience or BioLegend). To detect IAV-specific donor polyclonal CD4 T cells in adoptive hosts, cells were stained for $1 \mathrm{~h}$ at $37^{\circ} \mathrm{C}$ with a $1: 100$ dilution of $\mathrm{I}-\mathrm{A}^{\mathrm{b}} / \mathrm{NP}_{311-325}$-fluorochromelabelled tetramer obtained from Trudeau Institute prior to surface marker staining. Tetrameter-positive cells in the CD90.1 $1^{+} \mathrm{CD} 90.2^{-} \mathrm{CD} 4^{+} \mathrm{CFSE} \mathrm{E}^{\text {low }}$ gate were enumerated.

Intracellular cytokine staining was performed as previously described ${ }^{42}$. In brief, cells were stimulated for $16 \mathrm{~h}$ with OVA peptide-pulsed APC. After $2 \mathrm{~h}, 10 \mu \mathrm{g} \mathrm{ml}{ }^{-1}$ Brefeldin A (Sigma) was added. Cells were then surface stained, fixed for $20 \mathrm{~min}$ in $4 \%$ paraformaldehyde, and permeablized by $10 \mathrm{~min}$ incubation in $0.1 \%$ saponin before staining for cytokine by the addition of a 1:100 dilution of anti-IFN- $\gamma$, TNF and IL-2, fluorescently labelled Abs. Intracellular staining for Bim (Cell Signaling Technology) and Bcl-2 (BD Pharmingen) was performed as per the manufacturer's instructions. Analysis was performed using LSRII instruments (BD Biosciences) and FlowJo (Tree Star) analysis software.

Detection of secreted cytokines. Naive or primed DO11.10 CD4 T cells, or donor DO11.10 populations isolated from IAV-challenged hosts, and syngeneic irradiated APC were cultured together at a 1:1 ratio with or without various concentrations of $\mathrm{OVA}_{323-339}$ peptide for $18 \mathrm{~h}$ or with $1 \mu \mathrm{g}$ of OVA peptide for various periods of time. Culture supernatants were harvested from triplicate wells and ELISA assays performed as per the manufacturer's instructions to determine levels of IFN- $\gamma$ and IL-2 (eBioscience).

Isolation of donor memory cells for microarray data analysis. WT or $I l 2^{-1-}$ D011.10 memory cells, $1 \times 10^{6}$, were CFSE-labelled and transferred to naive hosts then infected with PR8-OVA $\mathrm{II}$. Donor cells that had undergone $>5$ divisions were sort-purified from lungs at 7 d.p.i. and total mRNA isolated (Qiagen) and amplified (MessageAmp; Ambion). cRNAs were labelled and hybridized to M430 2.0 chips according to Affymetrix protocols. Data were normalized with the Plier algorithm, Log transformed and analysed with GeneSpring GX 11.0 (Agilent Technologies). Significant genes were selected based on $P$ values $<0.05$ and fold change $>1.5$ or 2 after application of the Benjamini and Hochberg correction for false discovery. Heat maps were generated using Gene-E (The Broad Institute).

Statistical analysis. Group sizes of $n=3-5$ were employed. Unpaired, two-tailed, Student's $t$-tests, $\propto=0.05$, were used to assess whether the means of two normally distributed groups differed significantly. One-way ANOVA analysis with Bonferroni's multiple comparison post-test was employed to compare multiple means. All error bars represent the s.d. Significance is indicated as ${ }^{\star} P<0.05,{ }^{* *} P<0.005$ ${ }^{* * *} P<0.001,{ }^{* * * *} P<0.0001$.

\section{References}

1. Kaech, S. M., Wherry, E. J. \& Ahmed, R. Effector and memory T-cell differentiation: implications for vaccine development. Nat. Rev. Immunol. 2, 251-262 (2002).

2. McKinstry, K. K., Strutt, T. M. \& Swain, S. L. Regulation of CD4+ T-cell contraction during pathogen challenge. Immunol. Rev. 236, 110-124 (2010).

3. Masopust, D., Kaech, S. M., Wherry, E. J. \& Ahmed, R. The role of programming in memory T-cell development. Curr. Opin. Immunol. 16, 217-225 (2004).

4. McKinstry, K. K. et al. Memory CD4 $+\mathrm{T}$ cells protect against influenza through multiple synergizing mechanisms. J. Clin. Invest. 122, 2847-2856 (2012).

5. Teijaro, J. R., Verhoeven, D., Page, C. A., Turner, D. \& Farber, D. L. Memory CD4 T cells direct protective responses to influenza virus in the lungs through helper-independent mechanisms. J. Virol. 84, 9217-9226 (2010).

6. Lenardo, M. et al. Mature T lymphocyte apoptosis-immune regulation in a dynamic and unpredictable antigenic environment. Annu. Rev. Immunol. 17, 221-253 (1999).

7. Williams, M. A., Tyznik, A. J. \& Bevan, M. J. Interleukin-2 signals during priming are required for secondary expansion of CD8 + memory T cells. Nature 441, 890-893 (2006).

8. Feau, S., Arens, R., Togher, S. \& Schoenberger, S. P. Autocrine IL-2 is required for secondary population expansion of CD8 $(+)$ memory T cells. Nat. Immunol. 12, 908-913 (2011).

9. Dooms, H., Wolslegel, K., Lin, P. \& Abbas, A. K. Interleukin-2 enhances $\mathrm{CD} 4+\mathrm{T}$ cell memory by promoting the generation of IL-7R alpha-expressing cells. J. Exp. Med. 204, 547-557 (2007).

10. Kalia, V. et al. Prolonged interleukin-2Ralpha expression on virus-specific $\mathrm{CD} 8+\mathrm{T}$ cells favors terminal-effector differentiation in vivo. Immunity 32, 91-103 (2010).

11. Blattman, J. N. et al. Therapeutic use of IL-2 to enhance antiviral T-cell responses in vivo. Nat. Med. 9, 540-547 (2003).

12. Snow, A. L., Pandiyan, P., Zheng, L., Krummey, S. M. \& Lenardo, M. J. The power and the promise of restimulation-induced cell death in human immune diseases. Immunol. Rev. 236, 68-82 (2010).

13. Zhang, X., Giangreco, L., Broome, H. E., Dargan, C. M. \& Swain, S. L. Control of CD4 effector fate: transforming growth factor beta 1 and interleukin 2 synergize to prevent apoptosis and promote effector expansion. J. Exp. Med. 182, 699-709 (1995)

14. Strutt, T. M., McKinstry, K. K., Kuang, Y., Bradley, L. M. \& Swain, S. L. Memory CD4 + T-cell-mediated protection depends on secondary effectors that are distinct from and superior to primary effectors. Proc. Natl Acad. Sci. USA 109, E2551-E2560 (2012).

15. Mizuochi, T., Tentori, L., Sharrow, S. O., Kruisbeek, A. M. \& Singer, A. Differentiation of Ia-reactive CD8 + murine $\mathrm{T}$ cells does not require Ia engagement. Implications for the role of CD4 and CD8 accessory molecules in T cell differentiation. J. Exp. Med. 168, 437-442 (1988).

16. Teixeiro, E. et al. Different $\mathrm{T}$ cell receptor signals determine CD8 + memory versus effector development. Science 323, 502-505 (2009)

17. Zehn, D., Lee, S. Y. \& Bevan, M. J. Complete but curtailed T-cell response to very low-affinity antigen. Nature 458, 211-214 (2009).

18. Lemos, M. P., Fan, L., Lo, D. \& Laufer, T. M. CD8alpha + and CD11b + dendritic cell-restricted MHC class II controls Th1 CD4 + T cell immunity. J. Immunol. 171, 5077-5084 (2003). 
19. Chapman, T. J., Castrucci, M. R., Padrick, R. C., Bradley, L. M. \& Topham, D. J. Antigen-specific and non-specific CD4 $+\mathrm{T}$ cell recruitment and proliferation during influenza infection. Virology 340, 296-306 (2005).

20. Malek, T. R. The biology of interleukin-2. Annu. Rev. Immunol. 26, 453-479 (2008).

21. Boyman, O. \& Sprent, J. The role of interleukin-2 during homeostasis and activation of the immune system. Nat. Rev. Immunol. 12, 180-190 (2012).

22. Smith, K. A. \& Popmihajlov, Z. The quantal theory of immunity and the interleukin-2-dependent negative feedback regulation of the immune response. Immunol. Rev. 224, 124-140 (2008).

23. Boyman, O., Kovar, M., Rubinstein, M. P., Surh, C. D. \& Sprent, J. Selective stimulation of $\mathrm{T}$ cell subsets with antibody-cytokine immune complexes. Science 311, 1924-1927 (2006).

24. Surh, C. D., Boyman, O., Purton, J. F. \& Sprent, J. Homeostasis of memory T cells. Immunol. Rev. 211, 154-163 (2006).

25. Li, J., Huston, G. \& Swain, S. L. IL-7 promotes the transition of CD4 effectors to persistent memory cells. J. Exp. Med. 198, 1807-1815 (2003).

26. Kondrack, R. M. et al. Interleukin 7 regulates the survival and generation of memory CD4 cells. J. Exp. Med. 198, 1797-1806 (2003).

27. Boyman, O., Ramsey, C., Kim, D. M., Sprent, J. \& Surh, C. D. IL-7/anti-IL-7 $\mathrm{mAb}$ complexes restore $\mathrm{T}$ cell development and induce homeostatic $\mathrm{T}$ Cell expansion without lymphopenia. J. Immunol. 180, 7265-7275 (2008).

28. Grabstein, K. H. et al. Inhibition of murine B and T lymphopoiesis in vivo by an anti-interleukin 7 monoclonal antibody. J. Exp. Med. 178, 257-264 (1993).

29. Marrack, P. \& Kappler, J. Control of T cell viability. Annu. Rev. Immunol. 22, 765-787 (2004).

30. Li, X., McKinstry, K. K., Swain, S. L. \& Dalton, D. K. IFN-gamma acts directly on activated CD4 $+\mathrm{T}$ cells during mycobacterial infection to promote apoptosis by inducing components of the intracellular apoptosis machinery and by inducing extracellular proapoptotic signals. J. Immunol. 179, 939-949 (2007).

31. Peperzak, V., Xiao, Y., Veraar, E. A. \& Borst, J. CD27 sustains survival of CTLs in virus-infected nonlymphoid tissue in mice by inducing autocrine IL-2 production. J. Clin. Invest. 120, 168-178 (2010).

32. Matter, M. S., Claus, C. \& Ochsenbein, A. F. CD4 + T cell help improves $\mathrm{CD} 8+\mathrm{T}$ cell memory by retained CD27 expression. Eur. J. Immunol. 38, 1847-1856 (2008).

33. Dolfi, D. V. et al. Late signals from CD27 prevent Fas-dependent apoptosis of primary CD8 + T cells. J. Immunol. 180, 2912-2921 (2008).

34. Penaloza-MacMaster, P. et al. Opposing effects of CD70 costimulation during acute and chronic lymphocytic choriomeningitis virus infection of mice. J. Virol. 85, 6168-6174 (2011).

35. Xiao, Y., Peperzak, V., Keller, A. M. \& Borst, J. CD27 instructs CD4 + T cells to provide help for the memory CD8 $+\mathrm{T}$ cell response after protein immunization. J. Immunol. 181, 1071-1082 (2008).

36. Ballesteros-Tato, A., Leon, B., Lund, F. E. \& Randall, T. D. Temporal changes in dendritic cell subsets, cross-priming and costimulation via CD70 control CD8(+) T cell responses to influenza. Nat. Immunol. 11, 216-224 (2010).

37. Kuka, M., Munitic, I., Giardino Torchia, M. L. \& Ashwell, J. D. CD70 is downregulated by interaction with CD27. J. Immunol. 191, 2282-2289 (2013).

38. van Gisbergen, K. P. et al. The costimulatory molecule CD27 maintains clonally diverse CD8 $(+) \mathrm{T}$ cell responses of low antigen affinity to protect against viral variants. Immunity 35, 97-108 (2011).

39. Sojka, D. K., Bruniquel, D., Schwartz, R. H. \& Singh, N. J. IL-2 secretion by $\mathrm{CD} 4+\mathrm{T}$ cells in vivo is rapid, transient, and influenced by TCR-specific competition. J. Immunol. 172, 6136-6143 (2004).
40. Keller, A. M. et al. Costimulatory ligand CD70 is delivered to the immunological synapse by shared intracellular trafficking with MHC class II molecules. Proc. Natl Acad. Sci. USA 104, 5989-5994 (2007).

41. Garrod, K. R. et al. Dissecting T cell contraction in vivo using a genetically encoded reporter of apoptosis. Cell Rep. 2, 1438-1447 (2012).

42. Roman, E. et al. CD4 effector T cell subsets in the response to influenza: heterogeneity, migration, and function. J. Exp. Med. 196, 957-968 (2002).

43. Wakim, L. M., Woodward-Davis, A. \& Bevan, M. J. Memory T cells persisting within the brain after local infection show functional adaptations to their tissue of residence. Proc. Natl Acad. Sci. USA 107, 17872-17879 (2010).

44. Divekar, A. A. et al. Protein vaccines induce uncommitted IL-2-secreting human and mouse CD4 T cells, whereas infections induce more IFN-gammasecreting cells. J. Immunol. 176, 1465-1473 (2006).

45. Farber, D. L., Yudanin, N. A. \& Restifo, N. P. Human memory T cells: generation, compartmentalization and homeostasis. Nat. Rev. Immunol. 14, 24-35 (2014)

46. Strutt, T. M. et al. Multipronged CD4 $(+)$ T-cell effector and memory responses cooperate to provide potent immunity against respiratory virus. Immunol. Rev. 255, 149-164 (2013).

47. Swain, S. L., McKinstry, K. K. \& Strutt, T. M. Expanding roles for CD4(+) $\mathrm{T}$ cells in immunity to viruses. Nat. Rev. Immunol. 12, 136-148 (2012).

48. Strutt, T. M. et al. Memory CD $4+\mathrm{T}$ cells induce innate responses independently of pathogen. Nat. Med. 16, 558-564 551p following 564 (2010).

49. McKinstry, K. K. et al. Rapid default transition of CD4 T cell effectors to functional memory cells. J. Exp. Med. 204, 2199-2211 (2007).

50. Lyons, A. B. \& Parish, C. R. Determination of lymphocyte division by flow cytometry. J. Immunol. Methods 171, 131-137 (1994).

\section{Acknowledgements}

The US National Institutes of Health (P01AI046530 and R01AI076534 to S.L.S.), American Heart Association (14SDG18600020 to K.K.M.), Trudeau Institute Inc., and the University of Massachusetts Medical School supported this work. We thank Drs R. Dutton, R. Welsh, F. Chan and K. Smith for thorough discussions and comments on the manuscript.

\section{Author contributions}

K.K.M., T.M.S. and S.L.S. designed the research and wrote the manuscript. K.K.M., T.M.S., B.B., W.Z. and Y.K. performed experiments. A.M.C. contributed reagents, key mouse strain and critical comments on the research.

\section{Additional information}

Accession codes: The data for the microarray analysis are deposited in the Gene Expression Omnibus, accession number GSE60186.

Supplementary Information accompanies this paper at http://www.nature.com/ naturecommunications

Competing financial interests: The authors declare no competing financial interest.

Reprints and permission information is available online at http://npg.nature.com/ reprintsandpermissions/

How to cite this article: McKinstry, K. K. et al. Effector CD4 T-cell transition to memory requires late cognate interactions that induce autocrine IL-2. Nat. Commun. 5:5377 doi: $10.1038 /$ ncomms6377 (2014) 